\title{
Inferring subtle stress changes related to blasting and large seismic events in mines using ambient noise
}

\author{
G Olivier Institute of Mine Seismology, Australia
}

\begin{abstract}
Over the last decade, using ambient seismic noise correlations has been widely adopted in crustal seismology to image and monitor the subsurface. The method relies on the reconstruction of the seismic Green's function between sensors, effectively turning one station in to a virtual active source. Since this process is repeatable, subtle changes in seismic velocity, attenuation and anisotropy can be measured over time. In some settings, these measurements can be made on an hourly (or even shorter) basis. To use this method routinely in underground mines, it is important to determine the cause of velocity variations in the absence of large dynamic stress perturbations (such as blasts). It also is important to calibrate the seismic velocity changes in terms of known stress changes so the effect of mining activities can be quantified in units that can be used by geotechnical engineers. The results presented here indicate that atmospheric air pressure changes can have a measurable influence on the long term seismic velocity variations at depth in the absence of large dynamic stress perturbations. This influence enabled me to determine the sensitivity of the relative velocity changes to stress, where a value of $3.2 \times 10^{-6} \% \mathrm{~Pa}$ was found. This calibration essentially enables me to turn each sensor pair in an underground mine into a stress meter, which in turn enabled me to infer the subtle change in static stress after a blast. The static stress change was only visible after the non-linear behaviour (damage and relaxation) had subsided five days after the blast. This new method can be used by geotechnical engineers to monitor the evolution of stress and to assess seismic hazard in conjunction with conventional microseismic methods.
\end{abstract}

Keywords: passive seismic, ambient noise, seismic monitoring

\section{Introduction}

Deep underground mines are highly stressed environments where seismic activity with following rockfalls can be dangerous. As a result, many mines have dense, modern seismic monitoring networks. Because almost all seismic events in mines are induced by mining activities, it is important for geotechnical engineers to monitor the consequences of mining activities on the rock mass over time. Over the past 20 years, seismic monitoring in mines has made great progress as a tool to analyse some of the risks involved in mining at depth (e.g. Riemer \& Durrheim 2011), but seismic activity alone is hard to interpret.

Seismic velocities of intact rock long have been known to be sensitive to the internal state of the rock mass. Changes in applied stress (Larose \& Hall 2009), pore pressure (Brenguier et al. 2014), and fracture density (Nur \& Simmons 1969; Nur 1971) all have been shown to cause measurable changes in seismic velocity. In particular, the relationship between seismic velocity and stress has been tempting researchers for many years to investigate time-dependent seismic velocity variations as a possible method to predict earthquakes.

Different types of controlled man-made seismic sources have been used to produce repetitive waveforms to measure seismic velocity variations (Fazio et al. 1973; Reasonberg \& Aki 1974; Yamamura et al. 2003). Alternatively, earthquake doublets have been used together with coda wave interferometry to exploit the high sensitivity of coda waves to small changes in seismic velocity (Poupinet et al. 1984; Snieder \& Hagerty 2004). Man-made seismic sources have the advantage that the initiation time and location of the seismic signal are controllable so that measurements can be made routinely, but these are expensive and have limited range. On the other hand, when using techniques that rely on uncontrolled seismic sources (e.g. earthquake doublet analysis), we have no control over the temporal resolution of our measurements or spatial distribution of sources. 
The seismic Green's function between two points represents the signal that would be recorded at one of these points if there was an impulsive source at the other point. Over the past decade, reconstructing the seismic Green's function between two seismic stations using ambient noise cross-correlations has emerged as a promising approach for exploring the earth's interior without the requirement of costly active seismic sources (Lobkis \& Weaver 2001; Campillo \& Paul 2003; Shapiro \& Campillo 2004). The cross-correlation function is often referred to as a virtual seismic source signal as opposed to the seismic Green's function, as it represents the signal that would be recorded at one station if the other station was replaced by a seismic source. This approach has been used to monitor velocity variations in large structures such as volcanoes (Brenguier et al. 2011), active fault zones (Brenguier et al. 2008a), and even across entire countries (Brenguier et al. 2014). In these studies, interstation distances range between tens of kilometres and thousands of kilometres.

Recently, the possibility of using this method in an industrial setting (with approximately $100 \mathrm{~m}$ interstation distances) was confirmed by Olivier et al. (2015a), in which the authors developed a processing scheme that succeeds in recovering estimates of the seismic Green's functions (including body-waves) between sensors located in an active underground mine by cross-correlating seismic noise generated by mining activities. The ability to reconstruct direct body-wave arrivals with correlations of seismic noise means that I can essentially turn each sensor into a virtual seismic source. With this virtual seismic source, I can create 3D velocity models of the underground mining area (Olivier et al. 2015a), which can potentially be used to locate seismic events more accurately than with homogeneous seismic velocity models. Additionally, these virtual seismic sources can be used for underground seismic exploration.

The possibility of accurately monitoring the change of seismic velocity with time may provide information on the response of the rock mass to mining activities. In turn, this may increase our ability to determine short and long-term seismic hazards in different sections in a mine. In this paper, I discuss the results of my attempts to use ambient seismic noise correlations to monitor subtle changes in seismic velocity in underground mines. The results shown here are a combination of results from three recent publications (Olivier et al. 2015a, 2015b; Oliver \& Brenguier 2016).

\section{Seismic velocities in rock}

Seismic velocities can either be measured by passive or active methods in the earth's crust. Active methods are inconvenient, sometimes very expensive and have limited range. On the other hand, most passive methods (like travel time tomography with passive seismic events) can only measure velocity changes accurately enough to detect relative velocity variations of the order of 1 percent and with temporal resolution on the order of one month (Patanè et al. 2006), which are not accurate or frequent enough to detect processes associated with earthquake nucleation or relaxation. Over the last decade, it has been shown that the use of ambient seismic noise can bridge the gap between active and passive methods - it can enable us to make daily (or better) measurements of seismic velocity variations over long ranges without any extra instrumentation (e.g. Brenguier et al. 2014).

In this section I will briefly discuss the two main parameters driving the seismic velocity changes in the earth's crust. The seismic velocities of rock are dependent on the elastic moduli, the stress (direction and magnitude) and fractures (density, orientation and saturation). For intact rock, only the stresses and fractures are likely to change. In this section I will briefly discuss the dependence of seismic velocity on these two parameters.

\subsection{Dependence on stress}

The dependence of seismic velocity on applied stress has long been known (e.g. Birch 1961; Nur \& Simmons 1969; Nur 1971; O'Connell \& Budiansky 1974; Lockner et al. 1977). This effect has been observed in a variety of laboratory (Eberhart-Phillips et al. 1989; Verdon et al. 2008; Larose \& Hall 2009) and field studies (Fazio et al. 1973; Reasonberg \& Aki 1974; Leary et al. 1979; Yamamura et al. 2003; Silver et al. 2007).

The dependence of the seismic velocity on stress differs for different rock types, but for a certain pressure range this relationship appears to be linear to a first-order approximation (Eberhart-Phillips et al. 1989; Verdon et al. 2008; Larose \& Hall 2009). There are two descriptions for the influence of changing stresses on 
seismic velocities. The first is the closing of microcracks which elastically stiffens the rock so that an increase in stress results in an increase in velocity and vice versa. This description is very intuitive, but hard to formulate and implement mathematically.

The second description comes directly from third-order elasticity theory (Murnaghan 1951), where the seismic velocities can be expressed in terms of isotropic strain and elastic moduli as:

$$
V_{p}=\sqrt{\frac{\lambda+2 \mu+\frac{1}{3}(7 \lambda+10 \mu+6 l+4 m) \theta}{\rho_{0}}}
$$

and

$$
V_{S}=\sqrt{\frac{\mu+\left(\lambda+\mu+m-\frac{n}{6}\right) \theta}{\rho_{0}}}
$$

where:

$\theta$ is the volumetric strain.

$\lambda$ and $\mu$ are the second-order elastic moduli (or Lamé's constants).

I, $\mathrm{m}$ and $\mathrm{n}$ are the third-order elastic moduli (or Murnaghan constants).

The difference between the expressions for the $\mathrm{P}$ and $\mathrm{S}$-wave velocities shown here and the ones more often used are from the contributions of the third-order terms of the strain energy function, which are generally neglected. As shown in Aoki (2015) in the absence of damage from dynamic stress perturbations, change in seismic velocity due to infinitesimal change in isotropic strain can be approximated by:

$$
\frac{1}{V_{p}} \frac{d V_{p}}{d \theta}=\frac{7 \lambda+10 \mu+6 l+4 m}{6(\lambda+2 \mu)}
$$

and

$$
\frac{1}{V_{s}} \frac{d V_{S}}{d \theta}=2+\frac{\lambda+m-n / 6}{2 \mu}
$$

This description can be used relatively easily to estimate the response of the seismic velocities to strain changes, but no physical interpretation of the moduli have been formulated so that their values are hard to estimate (Aoki 2015). Tsai (2011) modelled the expected velocity change from the thermoelastic and poroelastic strain changes with reasonable values of the third-order elastic constants to investigate if seasonal variations in seismic velocity observed by Meier et al. (2010) in southern California can be explained by this theory. Here the author found that the observed velocity variations are generally too large to be explained by third-order elasticity theory so that another mechanism was probably responsible for the velocity variations. Although it is hard to determine which one of the two descriptions best describes the susceptibility of seismic velocity to stress change, knowing the exact mechanism is not strictly necessary for the purposes of this paper.

The influence of stress on seismic velocity, along with the fact that earthquakes are initiated by accumulating tectonic stress, has for a long time lead researchers to believe that accurate measurement of time-dependent seismic velocity variations could potentially be a method to predict earthquakes (Whitcomb et al. 1973; Mjachkin et al. 1975). Seismic velocity variations have been reported prior to a few large earthquakes (Niu et al. 2008; Lucente et al. 2010), but more results are needed for the correlation to be convincing.

Currently stress meters, borehole strain cells, gauges, etc. are used in mines to determine the values of stress and deformation in different areas in the mine (Fairhurst 2003). However, many of these measurements are very localised in space and can be plagued by site effects. Additionally, most of these methods are not practical to implement regularly. 
Although seismic velocities of rock are sensitive to applied stress, the susceptibility of seismic velocity to stress change is very low, i.e. of the order of $10^{-6} \% / \mathrm{Pa}$ (see table in Yamamura et al. 2003). Therefore, in order to measure $\mathrm{kPa}$ level stress changes, seismic velocities need to be measured with up to $0.001 \%$ accuracy. Some studies have attempted to measure the changes in seismic velocity due to changes in stress with microseismic events (Maxwell \& Young 1992; Westman et al. 2012) or active sources (Grêt et al. 2006; Huang et al. 2013) to evaluate the response of the rock to mining activities, but have not been able to reach the desired level of accuracy.

If virtual seismic sources can be used to regularly measure small changes in seismic velocity, it can provide information about how stresses are evolving as a function of time. Since the rate of microseismic activity in mines can be controlled to a certain degree (Mendecki 1997), it could be possible to evaluate the evolution of stress and plan mining activities accordingly and ultimately improve safety.

\subsection{Dependence on damage}

The linear relation between stress and seismic velocity is not always valid. In the presence of a sudden dynamic stress perturbation, like an earthquake or a blast, the rock responds in a very complex and non-linear fashion. In this scenario, the dynamic stress perturbation causes a sudden decrease in velocity followed by gradual recovery. These observations have been made in laboratory studies and is referred to as anomalous nonlinear fast dynamics and slow dynamics (Johnson \& Sutin 2005). This has also been observed in numerous crustal studies, specifically in relation to damage from large earthquakes (Brenguier et al. 2008a; Wegler et al. 2009; Cheng et al. 2010; Taira et al. 2015).

Damage here could refer to motions at grain joints or the opening of microfractures. Recently, non-linear damage rheology models have been developed to explain such behaviour (Lyakhovsky et al. 2009). It is also possible that the short-term velocity decrease prior to volcanoes are related to this non-linear effect (Brenguier et al. 2011). Both the linear and non-linear behaviour of rock in response to stress changes are of interest in a mining environment. For example, accurate measurements of damage induced by development blasts or induced earthquakes, a non-linear behaviour, can help determine the influence range of these events and time-dependent relaxation can be used to determine re-entry times. Measurements of stress change, a linear behaviour, can be used in conjunction with conventional passive seismic monitoring to determine seismic hazard as a function of space and time.

\section{Data and methods}

With steady advances in the computer technology, it has now become feasible to record a large amount of continuous seismic data from underground microseismic monitoring networks with high sampling rates for many mines. In this section I will show how this continuous seismic data can be used to turn seismic sensors into virtual sources.

\subsection{Garpenberg mine}

Continuous seismic data were recorded for one month (June 2013) at Boliden's Garpenberg mine, in Sweden, using the standard Institute of Mine Seismology seismic monitoring network. Garpenberg is a zinc, copper, lead, silver, and gold-producing mine. The seismic monitoring network consisted of 18 geophones $(14 \mathrm{~Hz})$ located approximately $1 \mathrm{~km}$ below surface. To avoid the zones of fractured rock around the mining tunnels, the geophones were cemented in boreholes $5 \mathrm{~m}$ away from the tunnels. The continuous seismic data were sampled at 6,000 samples per second and time synchronised by dedicated fibre optic cables from the central GPS receiver to each of the underground stations. The locations of the geophones relative to the mining tunnels are shown in Figure 1. 


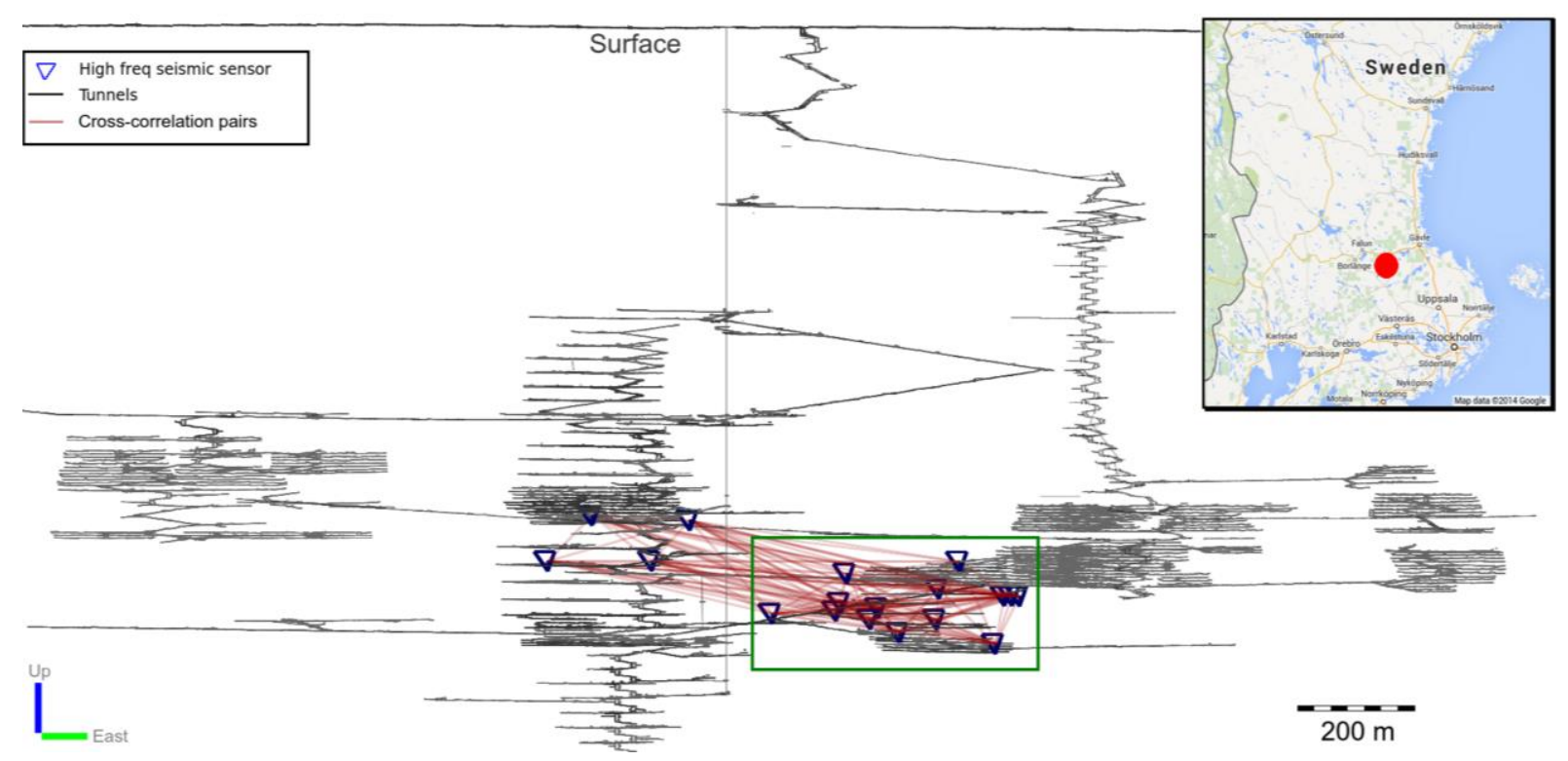

Figure 1 Section view of Garpenberg mine. The seismic sensors are indicated by blue triangles and the tunnel by grey lines. The cross-correlation pairs are indicated by red lines. The main study area is indicated by a green box

\subsection{Constructing virtual source signals from noise}

Almost 50 years ago, Claerbout argued that "by cross-correlating noise traces recorded at two locations on the surface, we can construct the wave field that would be recorded at one of the locations if there was a source at the other" (Claerbout 1968). Claerbout's conjecture was well before its time and only gained momentum in the seismology community a few decades later after the approach was successfully applied to helioseismic data (Duvall et al. 1996). A few years later Lobkis and Weaver (2001) showed that the Green's function emerges between two ultrasonic sensors by cross-correlating the signal recorded with them in the presence of a diffuse ultrasonic field. Here the authors remarked that equipartition of modes or a fully diffuse wavefield is required to retrieve the Green's function. This is a condition that is relatively easy to fulfil in ultrasonic experiments, but not realistic in seismology. Derode et al. (2003) showed that the Green's function can be retrieved by cross-correlation even in the absence of diffusivity with an argument based on time-reversal symmetry. However, the time-reversal symmetry is not valid in the presence of attenuation. Wapenaar (2004) used a reciprocity theorem to show that the Green's function can be retrieved in any inhomogeneous medium by cross-correlating the recordings of two sensors located at a free surface, also in the absence of diffusivity. However, this derivation required the receivers to be located at the surface.

Snieder (2004) showed that in a homogeneous elastic medium with scatterers that act as secondary sources, one can retrieve the Green's function by cross-correlating the signals recorded in at two receivers with the stationary phase approximation. This derivation did not require the receivers to be located at the surface and applied to any 3D distribution of sensors. Since then there have been numerous authors who have shown mathematically that the Green's function can emerge from seismic noise recordings (for a thorough review of these derivations and a discussion of their similarity see Boschi and Weemstra (2015).

The pre-processing steps to construct virtual sources from ambient seismic wavefields have become reasonably standard over the years (e.g. Lecocq et al. 2014). However some additional steps are required in the presence of non-stable seismic sources. In particular, the use of a selection filter is essential to construct stable virtual sources with seismic noise that exhibited spatio-temporal variations (Olivier et al. 2015b; Nakata et al. 2015). A brief outline of the steps is given below and illustrated in Figure 2. 



(b) Cross-correlate
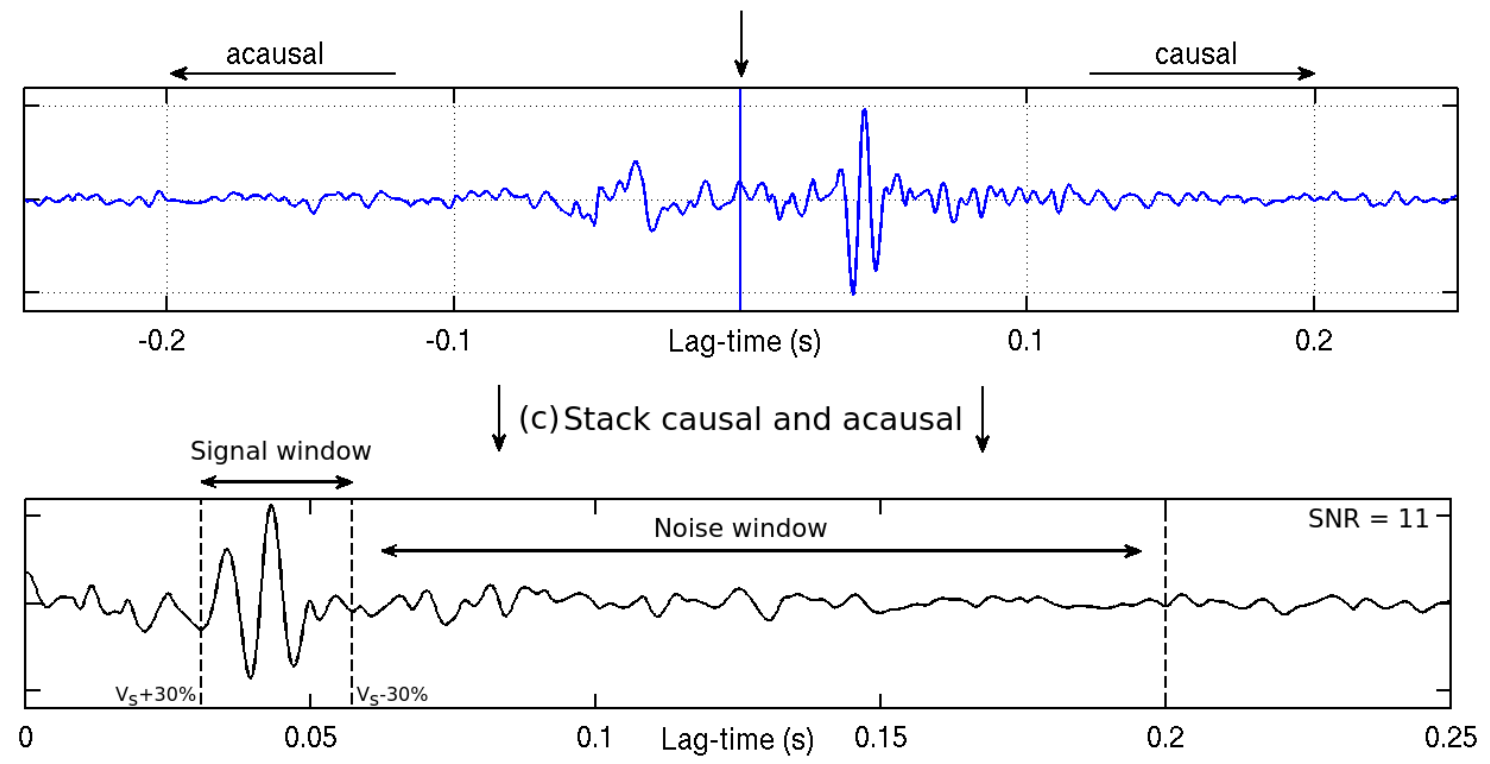

Figure 2 An example of the standard processing steps used to construct virtual source signals from continuous data sections

Olivier et al. (2015a) establish that the seismic noise recorded at Garpenberg can be used to reconstruct the seismic Green's functions between station pairs by using a modified processing scheme that selects periods of data based on the signal-to-noise ratio $(\mathrm{S} / \mathrm{N}$ ) of cross-correlation functions (CCFs). The processing steps are illustrated in Figure 1 and outlined below.

After the continuous seismic data for individual sensors are divided into short sections ( 0.5 to 20 s lengths), three main processing steps are required to enable us to reconstruct stable CCFs:

- The segments are spectrally whitened in a frequency range that is dependent on the seismic noise conditions. This process enhances the contributions of weak sources. For Garpenberg, the frequency range that produced the most stable results were between 20 and $400 \mathrm{~Hz}$. One-bit normalisation also is performed at this step in an attempt to further normalise the energy from different sources.

- Individual sections are cross-correlated between sensor pairs. For $\mathrm{N}$ sensors, there are $\mathrm{N}(\mathrm{N}-1) / 2$ sensor pairs.

- Finally, I calculate an S/N for each individual CCF as follows: The causal and acausal parts of the CCFs are summed, and the $\mathrm{S} / \mathrm{N}$ is calculated as the root mean square $(\mathrm{rms})$ of the summed CCF in a window $d W_{s}+30 \%$ to $d W_{s}-30 \%$ divided by the rms in the coda (after $d W_{s}-30 \%$ up to $0.4 \mathrm{~s}$ ) of the CCF, where $d$ is the distance between sensors and $V_{s}$ is the known $\mathrm{S}$-wave velocity as determined by calibration blasts (Olivier et al. 2015a). The causal and acausal parts are summed to potentially increase the $\mathrm{S} / \mathrm{N}$. 
In Figure 2, a S/N of 11 is obtained for an individual CCF. Individual CCFs are added to a stack for a station only if the $\mathrm{S} / \mathrm{N}$ exceeds a preselected minimum. The filter is intended to exclude periods of time when seismic noise is not propagating in stationary phase locations or when the seismic noise is dominated by monochromatic sources.

The specific values given in the steps above apply to the conditions at Garpenberg mine. The frequency range, $\mathrm{S} / \mathrm{N}$ minimum, time section lengths and even the use of one-bit normalisation is dependent on the local seismic noise and scattering conditions and require a thorough investigation to find the optimal parameters.

If any changes in seismic velocity or attenuation have occurred between a virtual source and a sensor, it will cause a small change in the virtual source signal. In the absence of any of these changes, the virtual source signals should remain identical over time.

\subsection{Measuring changes in seismic velocity}

Changes in seismic velocity are typically measured between a reference virtual seismic source signal and a current virtual source signal. Typically, only the coda (or tail) of the virtual source signal is considered when this measurement is made and apparent changes in the direct arrivals are neglected. This is done for two reasons. Firstly, the coda part of the virtual source signal is more robust than the direct arrival with regard to changes of the seismic noise sources (e.g. Froment et al. 2010; Colombi et al. 2014). Therefore, ignoring the direct arrival reduces the error introduced by changes in seismic noise sources. Secondly, the coda consists of scattered waves that sample the medium for a longer period and therefore small changes in velocity become clear in the coda even when no change can be measured in the direct arrivals.

This is illustrated in Figure 3, where an example of two cross-correlation functions that were made from data recorded a few days apart at Garpenberg are shown. A small change in seismic velocity is visible in the coda of the CCF, whereas the direct arrival appears to be unchanged. Also note that the two signals look very similar even though they were constructed using data that are separated by a few days, indicating the robustness of the method. The windows typically used to measure the changes in velocity are taken a few periods after the direct S-wave arrival, up to lag-times where the coda is stable (typically 0.2 to $2 \mathrm{~s}$ after the direct S-wave arrival). The changes that are measured in the coda mostly represent changes in shear-wave velocity (Snieder 2006).



Figure 3 Two CCFs for the same station pair constructed from data a few days apart shown in red and blue. There is no clear change in the direct arrival of the CCF (bottom left) since there is no separation between the blue and red lines, whereas a small travel time shift is noticeable in the coda part (bottom right) since the blue and red lines are slightly shifted 
Calculating the relative velocity changes is done by either using the doublet (Poupinet et al. 1984; Clarke et al. 2011; Lecocq et al. 2014) or stretching (Daskalakis 2016) method. The doublet technique first measures the time delay of the signal within sliding time-windows along the coda of the virtual source signal by analysing phase changes in the frequency domain. Each time-delay corresponds to a cross-correlation lag-time which is taken at the centre of the measuring time-window. A linear regression line is then fitted though the measured travel time variations, and the slope of this line provides an approximate estimate of the homogeneous velocity change through the medium. The stretching technique operates completely in time domain and effectively finds the stretching (or contraction) parameter that maximises the correlation coefficient between the daily and the reference virtual source signals.

Under ideal circumstances these methods give the same result, but in some cases one of these methods might be better suited than the other. The stretching method is more appropriate to use than the doublet method when large fluctuations in seismic velocity are expected, since the doublet method can underestimate large changes in seismic velocity due to period skipping in the coda of the correlation functions. The doublet method is more suitable when very small changes in seismic velocity are expected and in cases where the waveforms are not coherent over time due to strong changes in the seismic noise field.

In most cases in underground mines, the doublet method is the preferred way to measure changes in seismic velocity, since large changes are not expected. A notable exception has recently been shown in block cave mines, where large changes in travel time occur as the cave propagates between sensor pairs (Dales et al. 2016). Due to the large changes induced by the propagation of the block cave between sensors, the stretching method would be more applicable.

\section{$4 \quad$ Results}

Ambient seismic noise correlations are used in a number of deep mines around the world to measure changes in seismic velocity. In this section, I will show results from the first applications where seismic velocity changes were measured with ambient seismic noise correlation at Garpenberg mine.

\subsection{Background seismic velocity changes}

As stated in Section 2, two factors that influence seismic velocities are stress and damage. In order for seismic velocity variations to be useful for geotechnical engineers, it is important to calibrate the velocity changes in terms of known stress changes during times when the seismic velocity changes are driven by stress changes. In the absence of large stress perturbations like blasting or earthquakes, the seismic velocities should be linearly related to applied stress (to first-order).

To examine this, I considered the velocity variations averaged for all station pairs in Figure 4 during the first 15 days, where there were no large dynamic stress perturbations (blasts or large events). I compared it to a range of factors that have been known to influence the seismic velocity variations, namely: tidal strain, thermo elastic strain from surface temperature changes and atmospheric air pressure. By averaging all station pairs, the local effects driving seismic velocity change destructively interfere and the global effects stack constructively, improving our chances to detect velocity changes due to global stress changes. 




Figure 4 Seismic velocity variations averaged for all 153 station pairs at Garpenberg mine for the month of June 2013 (top) and seismic events recorded every two hours (bottom). During the first 15 days, no large events or blasting are evident, while a blast is indicated by a red dotted line on the 17th

Since the different factors that can influence seismic velocity changes act on different time scales, I divide the velocity variations in to long-term variations (green line in Figure 4) and short-term variations (blue line minus green line in Figure 4).

\subsubsection{Short-term velocity variations}

In Figure 5, I show the short-term variations (STV) in seismic velocity (averaged for all sensor pairs) during a relatively quiet period of time in the mine compared with the volumetric tidal deformations (modelled with GOTIC2 by Matsumoto et al. 2001) and temperature changes obtained from the nearest weather station at Hedemora. According to the data from the weather station, no significant rainfall occurred during this period. The changes in seismic velocity show no clear dependence on temperature or modelled tidal strain, with normalised correlation coefficients of 0.20 and 0.21 , respectively. The thermoelastic strain changes due to changes in temperature at the surface are delayed and constrained in depth by the fractured layer close to surface (Ben-Zion \& Leary 1986). This indicates that the STV due to temperature changes at surface are not straightforward at $1 \mathrm{~km}$ depth.
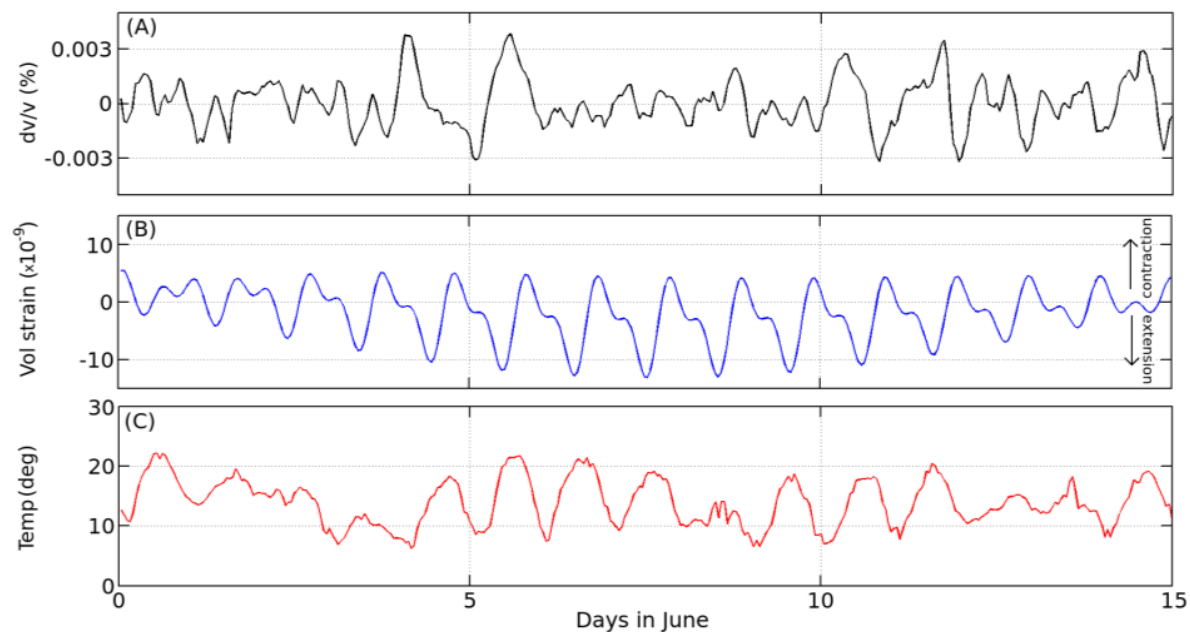

Figure 5 (a) Comparison of the short-term seismic velocity changes with (b) modelled volumetric tidal strain and (c) surface temperature changes. Neither of these parameters have an obvious influence on the seismic velocities 
To determine if these variations cause measurable effects at depth, a more in-depth statistical approach can be performed (Hillers et al. 2015). The time-dependent seismic velocity variations exhibit STV with large amplitudes. These could be related to mining activities, which can constantly change the state of the rock. However, these changes also may be related to variations in noise source locations which in turn depend on the current mining locations. The exact cause of the short-term velocity variations is still being investigated.

\subsubsection{Long-term velocity variations}

The LTV compared to atmospheric air pressure changes are shown in Figure 6. It appears as if the velocity variations are positively correlated and directly in phase with the changes in atmospheric air pressure, with a normalised correlation coefficient of 0.92 . I believe it is likely that the long-term measurements of seismic velocity change are more stable because the effects of day-to-day mining activities and their influence on seismic source distribution are removed by smoothing the time-dependent velocity variations. If I divide the long-term seismic velocity changes by the atmospheric pressure changes for each hour and calculate the median of these values, I get a sensitivity of velocity change to stress change of $3.2 \times 10^{-6} / \mathrm{Pa}$. This value compares well with the values reported in the literature (Yamamura et al. 2003). Although the changes in atmospheric air pressure are measured at surface, I estimate that the changes recorded at surface are approximately the same as at $1 \mathrm{~km}$ depth and experience no time delay (unlike thermoelastic strain changes). As noted by Hillers et al. (2015), the pressure induced stress changes propagate at shear-wave speed, which causes almost instantaneous changes at $1 \mathrm{~km}$ depth. This is similar to the estimate and observation made by Niu et al. (2008).


Figure 6 Comparison of the long-term seismic velocity changes with atmospheric air pressure changes. There appears to be a direct positive correlation between the two parameters, with a normalised correlation coefficient of 0.92

\subsection{Changes in seismic velocity during a blasting}

Olivier et al. (2015b) argue that the relative seismic velocity variations shown in Figure 5 showed the effect of two different mechanisms of seismic velocity change: (1) During and shortly after the blast, the seismic velocity changes are dominated by the damage and relaxation of the rock mass caused by the strong dynamic stress perturbations from the blast, and (2) in some areas (see red and blue traces in Figure 7), the seismic velocity does not appear to return to the previous values and a permanent change in velocity is observed. These permanent variations may be due to several factors, including anelastic changes in the rock due to the strong shaking from the blast and permanent changes in the static stress field and changes in the location and/or frequency of the noise sources (for instance aftershocks following the blast). However, the potential 
influence of changes in location and/or frequency of noise sources were previously reduced by averaging the velocity variations for each sensor because different sensor pairs are orientated differently to potential source changes (azimuthal averaging). Additionally, there were no noticeable changes in the similarity of the CCFs (decoherence) after the blast, and the seismic activity rate returned to normal shortly after the blast whereas the velocity changes remained clearly visible well after. The anelastic effects due to the blast may well have caused permanent changes in the rock mass (especially close to the blast), but to the best of my knowledge, these effects can only cause a decrease in seismic velocity.



Figure 7 Changes in seismic velocity for three seismic stations during a blast. At the time of the blast, a sudden decrease in seismic velocity followed by a slow relaxation (over five days) can be seen. For some stations (blue and red), the seismic velocities don't return to the same levels they were prior to the blast. The location of the blast relative to the sensors are shown in the inset

Because there are notable increases in the seismic velocity after the blast in some areas, I infer that the permanent changes in velocity resulting from the blast are at least partially due to the redistribution of stress from the removal of a section of load-bearing solid rock. This was confirmed by comparing the spatial distribution of the seismic velocity changes with the expected changes in volumetric stress from elastic static stress modelling. A spatial comparison of the permanent changes in seismic velocity and the expected static volumetric stress changes are shown in Figure 8. For more details on the static stress modelling, see Olivier et al. (2015b). 


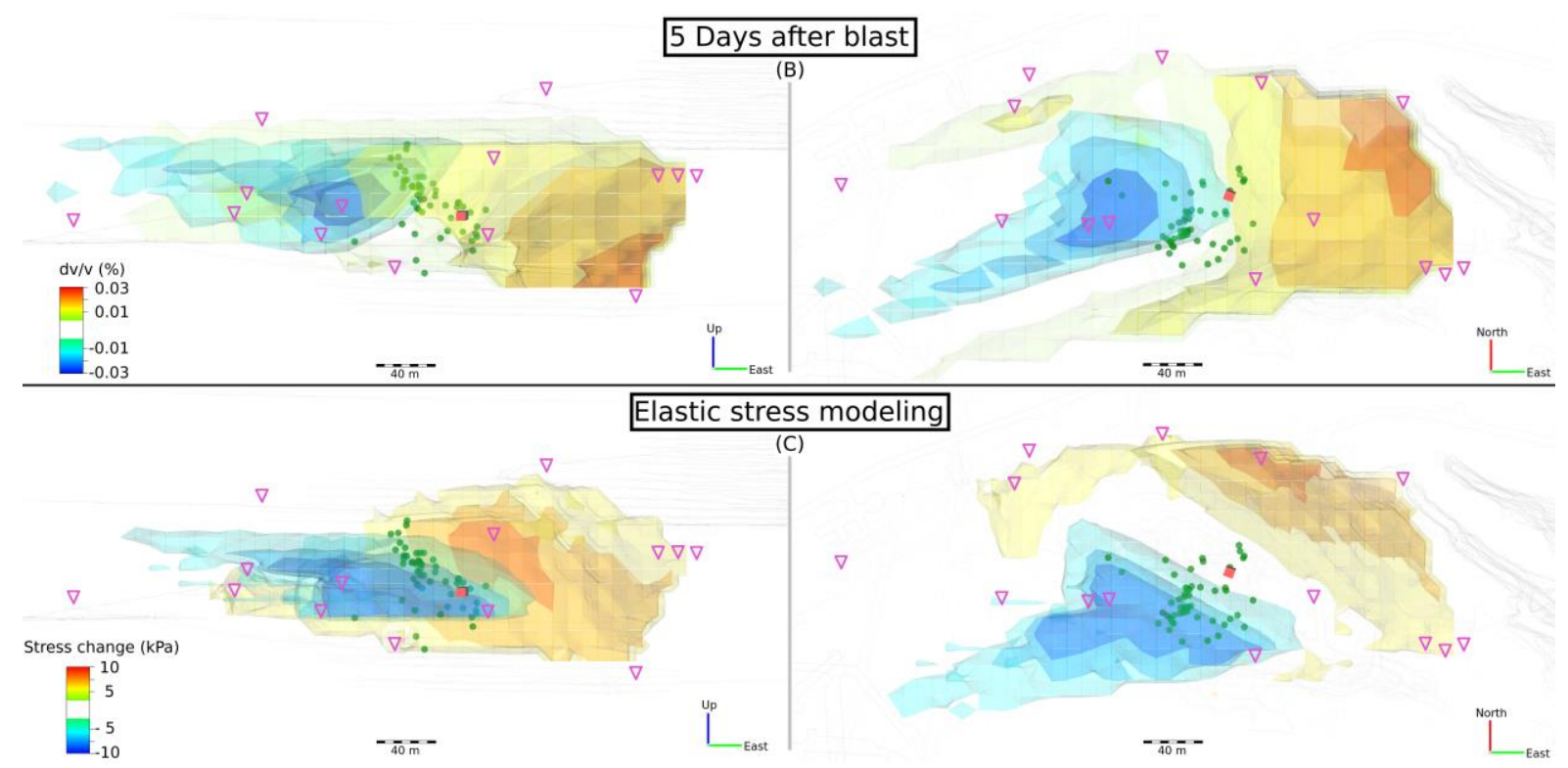

Figure 8 The spatial distribution of permanent velocity changes five days after the blast (top) with results from elastic static stress modelling (bottom). The two independent observations are generally in agreement. The location of the blast is shown in red and the locations of the aftershocks are shown in green spheres

\section{Conclusion}

In this paper, I reported on the recent progress that has been made in using ambient seismic noise cross-correlations to continuously monitor seismic velocity variations in underground mines.

I investigated the cause of the background fluctuations of the seismic velocity changes by comparing the changes to atmospheric air pressure variations, modelled tidal strain, and temperature changes at surface. By averaging the relative velocity variations of all 153 station pairs and separating the short and long-term seismic velocity changes, I established that the long-term fluctuations in seismic velocity are directly related to changes in atmospheric air pressure at surface. The relative seismic velocity sensitivity to changes in atmospheric air pressure was determined to be on average $3.2 \times 10^{-6} \% / \mathrm{Pa}$, which compares well with values reported in the literature. The origin of the short-term velocity variations does not appear to be due to temperature changes or earth tides, and I believe that the local mining activities are the dominant cause of the short-term fluctuations.

After calibrating the seismic velocity change in terms of atmospheric air pressure changes, I investigated the effects of a blast on the seismic velocities. The results indicate that the long-term behaviour of the seismic velocities is driven by subtle changes in static stress, whereas the short-term behaviour of the seismic velocity variations is due to the damage induced by the strong shaking from the blast.

Although the results shown here are related to blasting, I am confident that the same method can be applied to large seismic events in mines $(\mathrm{Mw}>1)$ and numerous experiments are currently underway.

This paper highlights the possibility of using ambient seismic noise to monitor the evolution of stresses in a mine. By removing the changes due to atmospheric air pressure fluctuations and calibrating the velocity changes in terms of known stress variations, I can essentially turn each seismic sensor into a stress meter. This can potentially be used as a standard monitoring method (in conjunction with conventional microseismic methods) and to evaluate seismic hazard in mines, ultimately improving safety.

\section{Acknowledgement}

I thank my collaborators Florent Brenguier, Richard Lynch, Michel Campillo, Philippe Roux and Nikolai Shapiro. I also thank all mine operators that allowed me to show these results. 


\section{References}

Aoki, Y 2015, 'Monitoring temporal changes of seismic properties', Frontiers in Earth Science, vol. 3, no. 42, article 42.

Ben-Zion, Y \& Leary, P 1986, 'Thermoelastic strain in a half-space covered by unconsolidated material', Bulletin of the Seismological Society of America, vol. 76, pp. 1447-1460.

Birch, F 1961, 'The velocity of compressional waves in rocks to 10 kilobars: 2', Journal of Geophysical Research, vol. 66 no. 7 , pp. 2199-2224.

Boschi, L \& Weemstra, C 2015, 'Stationary-phase integrals in the cross-correlation of ambient noise', Reviews of Geophysics, vol. 53, no. 2, pp. 411-451.

Brenguier, F, Campillo, M, Hadziioannou, C, Shapiro, N, Nadeau, R \& Larose, E 2008, 'Postseismic relaxation along the San Andreas fault at Parkfield from continuous seismological observations', Science, vol. 321, pp. 1478-1481.

Brenguier, F, Campillo, M, Takeda, T, Aoki, Y, Shapiro, N, Briand, X, Emoto, K \& Miyake, H 2014, 'Mapping pressurized volcanic fluids from induced crustal seismic velocity drops', Science, vol. 345, no. 6,192, pp. 80-82.

Brenguier, F, Clarke, D, Aoki, Y, Shapiro, N, Campillo, M \& Ferrazzini, V 2011, 'Monitoring volcanoes using seismic noise correlations', Comptes Rendus Geoscience, vol. 343, pp. 633-638.

Campillo, M \& Paul, A 2003, 'Long-range correlations in the diffuse seismic coda', Science, vol. 299, doi: 10.1126/science.1078551, pp. 547-549.

Cheng, X, Niu, F \& Wang, B 2010, 'Coseismic velocity change in the rupture zone of the $2008 \mathrm{Mw} 7.9$ Wenchuan earthquake observed from ambient seismic noise', Bulletin of the Seismological Society of America, vol. 100, no. 5B, pp. 2539-2550.

Claerbout, JF 1968, 'Synthesis of a layered medium from its acoustic transmission response', Geophysics, vol. 33, pp. 264-269.

Clarke, D, Zaccarelli, L, Shapiro, N \& Brenguier, F 2011, 'Assessment of resolution and accuracy of the moving window cross spectral technique for monitoring crustal temporal variations using ambient seismic noise', Geophysical Journal International, vol. 1,365, pp. 867-882.

Colombi, A, Chaput, J, Brenguier, F, Hillers, G, Roux, P \& Campillo, M 2014, 'On the temporal stability of the coda of ambient noise correlations', Comptes Rendus Geoscience, vol. 346, no. 11, pp. 307-316.

Dales, P, Audet, P \& Olivier, G 2016, 'A novel seismic source detection and location algorithm implemented to improve the construction of seismic Green's functions from ambient noise in mines', American Geophysical Union Fall Meeting Abstracts $1,2566$.

Daskalakis, E, Evangelidis, CP, Garnier, J, Melis, NS, Papanicolaou, G, Tsogka, C 2016, 'Robust seismic velocity change estimation using ambient noise recordings', Geophysical Journal International, vol. 205, no. 3, pp. 1926-1936.

Derode, A, Larose, E, Campillo, M \& Fink, M 2003, 'How to estimate the Green's function for a heterogeneous medium between two passive sensors?', Applied Physics Letters, vol. 83, pp. 3054-3056.

Duvall, T, D'silva, S, Jefferies, S, Harvey, J \& Schou, J 1996, 'Downflows under sunspots detected by helioseismic tomography', Nature, vol. 379, no. 6562, pp. 235-237.

Eberhart-Phillips, D, Han, D-H \& Zoback, MD 1989, 'Empirical relationships among seismic velocity, effective pressure, porosity, and clay content in sandstone', Geophysics, vol. 54, no. 1, pp. 82-89.

Fairhurst, C 2003, 'Stress estimation in rock: a brief history and review', International Journal of Rock Mechanics and Mining Sciences, vol. 40, no. 7, pp. 957-973.

Fazio, TD, Aki, L \& Alba, K 1973, 'Solid earth tide and observed change in the in situ seismic velocity', Journal of Geophysical Research, vol. 78, pp. 1319-1322.

Froment, B, Campillo, M, Roux, P, Gouédard, P, Verdel, A \& Weaver, RL 2010, 'Estimation of the effect of nonisotropically distributed energy on the apparent arrival time in correlations', Geophysics, vol. 75, no. 5, pp. SA85-SA93.

Grêt, A, Snieder, R \& Özbay, U 2006, 'Monitoring in situ stress changes in a mining environment with coda wave interferometry', Geophysical Journal International, vol. 167, no. 2, pp. 504-508.

Hillers, G, Ben-Zion, Y, Campillo, M \& Zigone, D 2015, 'Seasonal variations of seismic velocities in the san jacinto fault area observed with ambient seismic noise', Geophysical Journal International, vol. 202, no. 2, pp. 920-932.

Huang, J, Reyes-Montes, J \& Young, R 2013, 'Passive three-dimensional microseismic imaging for mining-induced rock-mass degradation', RapidMiner: Data Mining Use Cases and Business Analytics Applications, Chapman \& Hall/CRC Press, pp. 135.

Johnson, P \& Sutin, A 2005, 'Slow dynamics and anomalous nonlinear fast dynamics in diverse solids', The Journal of the Acoustical Society of America, vol. 117, no. 1, pp. 124-130.

Larose, E \& Hall, S 2009, 'Monitoring stress related velocity variation in concrete with a $2 \times 10-5$ relative resolution using diffuse ultrasound', The Journal of the Acoustical Society of America, vol. 125, no. 4, pp. 1853-1856.

Leary, P, Malin, P, Phinney, R, Brocher, T \& Voncolln, R 1979, 'Systematic monitoring of millisecond travel time variations near Palmdale, California', Journal of Geophysical Research: Solid Earth (1978-2012), vol. 84, no. (B2), pp. 659-666.

Lecocq, T, Caudron C \& Brenguier, F 2014, 'MSNoise, a Python package for monitoring seismic velocity changes using ambient seismic noise', Seismological Research Letters, vol. 85, doi: 10.1785/0220130073, pp. 715-726

Lobkis, O \& Weaver, R 2001 'On the emergence of the Green's function in the correlation of a diffuse field', Journal of the Acoustical Society of America, vol. 110, pp. 311-317.

Lockner, D, Walsh, J \& Byerlee, J 1977, 'Changes in seismic velocity and attenuation during deformation of granite', Journal of Geophysical Research, vol. 82, no. 33, pp. 5374-5378.

Lucente, FP, De Gori, P, Margheriti, L, Piccinini, D, Di Bona, M, Chiarabba, C \& Agostinetti, NP 2010, 'Temporal variation of seismic velocity and anisotropy before the $2009 \mathrm{mw} 6.3$ L'aquila earthquake, Italy', Geology, vol. 38, no. 11, pp. 1015-1018. 
Lyakhovsky, V, Hamiel, Y, Ampuero, J-P \& Ben-Zion, Y 2009, 'Non-linear damage rheology and wave resonance in rocks', Geophysical Journal International, vol. 178, no. 2, pp. 910-920.

Matsumoto, K, Sato, T, Takanezawa, T \& Ooe, M 2001, 'GOTIC2: A program for computation of oceanic tidal loading effect', Journal of the Geodetic Society of Japan, vol. 47, doi: 10.11366/sokuchi1954.47.243, pp. 243-248.

Maxwell, S \& Young, R 1992, 'Sequential velocity imaging and microseismic monitoring of mining-induced stress change', Pure and Applied Geophysics, vol. 139, no. 3-4, pp. 421-447.

Meier, U, Shapiro, NM \& Brenguier, F 2010, 'Detecting seasonal variations in seismic velocities within Los Angeles basin from correlations of ambient seismic noise', Geophysical Journal International, vol. 181, no. 2, pp. 985-996.

Mendecki, AJ 1997, Seismic monitoring in mines, Springer Science \& Business Media, Netherlands.

Mjachkin, VI, Brace, WF, Sobolev, GA \& Dieterich, JH 1975, 'Two models for earthquake forerunners', in M Wyss (ed), Earthquake Prediction and Rock Mechanics, Springer Basel AG, Basel, Switzerland, pp. 169-181.

Murnaghan, FD 1951, Finite Deformation of an Elastic Solid, Dover Publications Inc., Mineola, New York.

Nakata, N, Chang, JP, Lawrence, JF \& Boué, P 2015, 'Body wave extraction and tomography at Long Beach, California, with ambient-noise interferometry', Journal of Geophysical Research: Solid Earth, vol. 120, no. 2, pp. 1159-1173.

Niu, F, Silver, PG, Daley, TM, Cheng, X \& Majer, EL 2008, 'Preseismic velocity changes observed from active source monitoring at the Parkfield SAFOD drill site', Nature, vol. 454, no. 7,201, pp. 204-208.

Nur, A 1971, 'Effects of stress on velocity anisotropy in rocks with cracks', Journal of Geophysical Research, vol. 76, no. 8, pp. 2022-2034.

Nur, A \& Simmons, G 1969, 'Stress-induced velocity anisotropy in rock: An experimental study', Journal of Geophysical Research, vol. 74, no. 27 , pp. 6667-6674.

O'Connell, RJ \& Budiansky, B 1974, 'Seismic velocities in dry and saturated cracked solids', Journal of Geophysical Research, vol. 79, no. 35, pp. 5412-5426.

Olivier, G, Brenguier, F, Campillo, M, Lynch, R \& Roux, P 2015a,' Body-wave reconstruction from ambient seismic noise correlations in an underground mine', Geophysics, vol. 80, no. 3, doi: 10.1190/geo2014-0299.1, pp. KS11-KS25.

Olivier, G, Brenguier, F, Campillo, M, Roux, P, Shapiro, N \& Lynch, R 2015b, 'Investigation of coseismic and postseismic processes using in situ measurements of seismic velocity variations in an underground mine', Geophysical Research Letters, vol. 42, doi: 10.1002/2015GL065975, pp. 9261-9269.

Olivier, G \& Brenguier, F 2016, 'Interpreting seismic velocity changes observed with ambient seismic noise correlations', Interpretation, vol. 4, no. 3, pp. SJ77-SJ85.

Patanè, D, Barberi, G, Cocina, O, De Gori, P \& Chiarabba, C 2006, 'Time-resolved seismic tomography detects magma intrusions at Mount Etna, Science, vol. 313, no. 5,788, pp. 821-823.

Poupinet, G, Ellsworth, WL \& Frechet, J 1984, 'Monitoring velocity variations in the crust using earthquake doublets: An application to the Calaveras Fault, California', Journal of Geophysical Research: Solid Earth, vol. 89, no. B7, pp. 5719-5731.

Reasonberg, P \& Aki, K 1974, 'A precise, continuous measurement of seismic velocity for monitoring in situ stress', Journal of Geophysical Research, vol. 79, pp. 399-406.

Riemer, K \& Durrheim, R 2011, 'Mining seismicity in the Witwatersrand Basin: monitoring, mechanisms and mitigation strategies in perspective', Journal of Rock Mechanics and Geotechnical Engineering, vol. 3, no. 3, pp. 250-259.

Shapiro, NM \& Campillo, M 2004, 'Emergence of broadband Rayleigh waves from correlations of the ambient seismic noise', Geophysical Research Letters, vol. 31, no. 7, L07614.

Silver, PG, Daley, TM, Niu, F \& Majer, EL 2007, 'Active source monitoring of cross-well seismic travel time for stress-induced changes', Bulletin of the Seismological Society of America, vol. 97, no. 1B, pp. 281-293.

Snieder, R 2004, 'Extracting the Green's function from the correlation of coda waves: A derivation based on stationary phase', Physics Review E, vol. 69, 046610.

Snieder, R 2006, 'The theory of coda wave interferometry', Pure and Applied Geophysics, vol. 163, no. 2, pp. $455-473$.

Snieder, R \& Hagerty, M 2004, 'Monitoring change in volcanic interiors using coda wave interferometry: Application to Arenal Volcano, Costa Rica', Geophysical Research Letters, vol. 31, no. 9, L09608.

Taira, T, Brenguier, F \& Kong, Q 2015, 'Ambient noise based monitoring of seismic velocity changes associated with the 2014 mw 6.0 South Napa earthquake', Geophysical Research Letters, vol. 42, no.17, pp. 6997-7004.

Tsai, VC 2011, 'A model for seasonal changes in gps positions and seismic wave speeds due to thermoelastic and hydrologic variations', Journal of Geophysical Research: Solid Earth (1978-2012), vol. 116, no. B4.

Verdon, JP, Angus, DA, Michael Kendall, J \& Hall, SA 2008, 'The effect of microstructure and nonlinear stress on anisotropic seismic velocities', Geophysics, vol. 73, no. (4), pp. D41-D51.

Wapenaar, K 2004, 'Retrieving the elastodynamic Green's function of an arbitrary inhomogeneous medium by cross-correlation', Physics Review Letters, vol. 93, 254301.

Wegler, U, Nakahara, H, Sens-Schönfelder, C, Korn, M \& Shiomi, K 2009, 'Sudden drop of seismic velocity after the 2004 mw 6.6 mid-Niigata earthquake, Japan, observed with passive image interferometry', Journal of Geophysical Research, vol. 114, no. B6.

Westman, E, Luxbacher, K \& Schafrik, S 2012, 'Passive seismic tomography for three-dimensional time-lapse imaging of mining-induced rock mass changes', The Leading Edge, vol. 31, no. 3, pp. 338-345.

Whitcomb, JH, Garmany, JD \& Anderson, DL 1973, 'Earthquake prediction: Variation of seismic velocities before the San Francisco earthquake', Science, vol. 180, no. 4086, pp. 632-635.

Yamamura, K, Sano, O, Utada, H, Takei, Y \& Nakao, S 2003, 'Long-term observation of in situ seismic velocity and attenuation', Journal of Geophysical Research, vol. 108, pp. 2317-2331. 